\title{
Photonic band gaps, defect characteristics, and waveguiding in two-dimensional disordered dielectric and metallic photonic crystals
}

\author{
Mehmet Bayindir,* E. Cubukcu, I. Bulu, T. Tut, and E. Ozbay \\ Department of Physics, Bilkent University, Bilkent, 06533 Ankara, Turkey \\ C. M. Soukoulis \\ Ames Laboratory and Department of Physics, Iowa State University, Ames, Iowa 50011 \\ (Received 24 April 2001; revised manuscript received 16 July 2001; published 17 October 2001)
}

\begin{abstract}
We experimentally investigated the influence of positional disorder on the photonic band gap, defect characteristics, and waveguiding in two-dimensional dielectric and metallic photonic crystals. Transmission measurements performed on the dielectric photonic crystals have shown a stop band even if a large amount of disorder was introduced to these structures. On the other hand, the photonic band gap of the metallic crystals was found to be very sensitive to disorder, while the metallicity gap was not affected significantly. We addressed how the transmission characteristics of a cavity were affected in the presence of weak disorder. Since the translational symmetry was broken by disorders, we measured different cavity frequencies when we generated defects at various locations. We also demonstrated the propagation of photons by hopping through coupled-cavity structures in both dielectric and metallic two-dimensional photonic crystals. Effects of weak disorder on guiding and bending of electromagnetic waves through the coupled-cavity waveguides were also investigated.
\end{abstract}

DOI: 10.1103/PhysRevB.64.195113

PACS number(s): 42.70.Qs, 42.60.Da, 74.80.-g, 71.15.Ap

\section{INTRODUCTION}

Photonic crystals are periodic dielectric and metallic structures in which the refractive index modulation gives rise to stop bands for electromagnetic (EM) waves within a certain frequency range in all directions. In recent years, photonic band gap (PBG) materials have attracted much attention, and many possible applications of such materials were proposed and demonstrated. ${ }^{1,2}$

PBG materials are considered as the photonic analogues of the semiconductors. Existence of photonic band gaps, ${ }^{3}$ localization of classical waves in disordered photonic systems, ${ }^{4}$ defect modes ${ }^{5}$ are reminiscent of what we observed in electronic case. For instance, it is well known that the amorphous semiconductors have similar band gaps compared to their crystalline counterparts. Similarly, the existence of photonic gaps does not require long range periodic order in photonic structures. ${ }^{6}$ Therefore, investigation of localization phenomena and disorder effects in PBG materials has a fundamental importance in pure and applied physics.

By introducing a defect into an otherwise perfect photonic crystal, it is possible to create localized EM mode inside the photonic band gap. Photons with certain wavelengths can locally be trapped inside the defect volume. Properties of the cavity modes are reminiscent of the impurity states in a semiconductor. ${ }^{5,7}$ In the past several years, researchers have proposed various applications based on cavity structures built around photonic crystals such as waveguides, ${ }^{8-13}$ lasers, ${ }^{14,15}$ fibers, ${ }^{16}$ splitters, ${ }^{17}$ switches, ${ }^{17-20}$ and resonant antennas. $^{21}$

PBG structures also provide a promising tool to control and manipulate the flow of EM waves in integrated optical devices. ${ }^{1}$ Therefore, there is a growing interest in developing photonic crystal-based waveguides which can efficiently guide and bend EM waves either along a line defect (a row of missing rods) ${ }^{8-12}$ or through coupled cavities. ${ }^{13}$ In the latter case, which is known as coupled-cavity waveguides (CCW's), ${ }^{13}$ the EM waves were tightly confined at each defect site, and photons can propagate via hopping due to interaction between the neighboring evanescent cavity modes. ${ }^{22,23,13}$ This type of propagation mechanism was already demonstrated experimentally in 1D (Ref. 24) and 3D (Refs. 13 and 25) dielectric photonic crystals.

Since unintentional positional disorders are always present in the PBG structures, it is important to address the influence of disorders on the photonic band gaps, cavity modes, waveguides, and waveguide bends. Previously, the disorder effects were investigated in one-,${ }^{26-28}$ two-,${ }^{29-36}$ and three-dimensional ${ }^{37-41}$ photonic structures. However, to our knowledge, there are not any complete experimental studies on investigation of positional disorder effects in 2D dielectric and metallic photonic crystals.

Although the employment of PBG structures made of dielectric materials have been successful in various applications, there are certain advantages of introducing metals to the photonic crystals. ${ }^{42-47}$ For instance, the metallic photonic crystals have a large forbidden gap that extends from zero frequency up to the plasma frequency. ${ }^{44,47}$ Moreover, the metallic PBG structures are almost perfect reflectors in low frequency regions. ${ }^{43}$

The paper is organized as follows. In Sec. II, we first describe the experimental setup and construction of photonic crystals. We then investigate the effect of disorders on the photonic band gaps in 2D dielectric and metallic photonic crystals. Localization length was derived from the transmission measurements. Influence of disorder on defect characteristics of single rod removed cavities is presented in Sec. III. In Sec IV, we demonstrate guiding and bending of EM waves through 2D dielectric and metallic CCW's, and investigate how the positional disorder affects the waveguiding phenomena in these structures. 

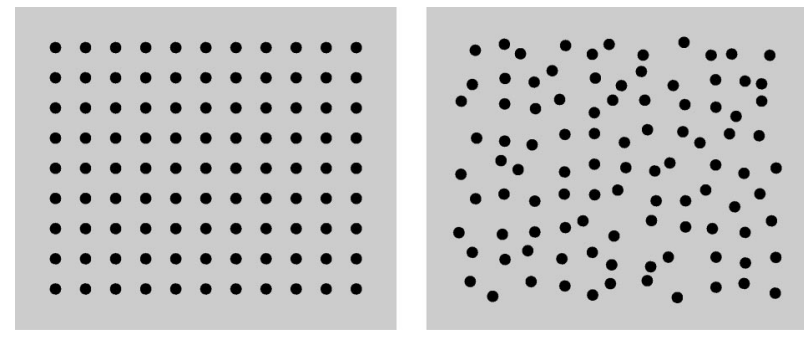

FIG. 1. Schematic drawing [top view] of the periodic (left panel) and disordered (right panel) two-dimensional photonic crystals. The disorder was introduced by changing the position of lattice points randomly.

\section{INFLUENCE OF DISORDER ON PHOTONIC BAND GAPS}

\section{A. Dielectric photonic crystals}

In our experiments, the dielectric photonic crystals were made up of cylindrical alumina rods having radius $1.55 \mathrm{~mm}$ and refractive index 3.1 at the microwave frequencies with a lattice constant $a=1.1 \mathrm{~cm}$. The experimental setup consisted of a HP 8510C network analyzer and microwave horn antennas to measure the transmission-amplitude spectra. We measured only transverse magnetic (TM) fields in which the incident electric field, E, was parallel to the rods. It is well known that the other polarization, transverse electric (TE), does not produce any photonic band gaps in this frequency range. 1

The positional disorder was introduced as follows. Each lattice point, $z_{i}$, was displaced according to $z_{i} \rightarrow z_{i}+r e^{i \varphi}$, where $r$ is the randomness parameter, and $\varphi$ is a random variable between $[0-2 \pi]$. Therefore, in this way, the degree of the disorder can be changed by varying the parameter $r$ between 0 and $a / 4$. Figure 1 shows the schematics of periodic (left panel) and the disordered (right panel) photonic crystals. In our experiments, we performed the transmission measurements by using five different random configurations for disordered case.

We first performed transmission measurements on the periodic and disordered dielectric crystals. We used two different values of the disorder parameter $r$, namely $r=a / 9$, and $a / 4$. The transmission was averaged over five different random configurations for the disordered case. For the periodic case, there appears a stop band extending from 8.67 to 13.25 $\mathrm{GHz}$ [Fig. 2(a), left panel]. When we introduced disorder, we observed that (1) the width of the stop band became narrower, and (2) the upper band edge decreased nearly $15 \mathrm{~dB}$. It was also observed that the photonic band gap persisted even if large amount of disorder was introduced [Figs. 2(b) and 2(c), left panel]. This observation is consistent with the argument that the long-range order is not necessary to achieve stop bands in dielectric photonic crystals. 6,35

We also considered frequency dependence of the localization length which is usually defined as $35,34,38$

$$
\xi^{-1}(\omega)=-\frac{\langle\ln T(\omega)\rangle}{2 L}
$$

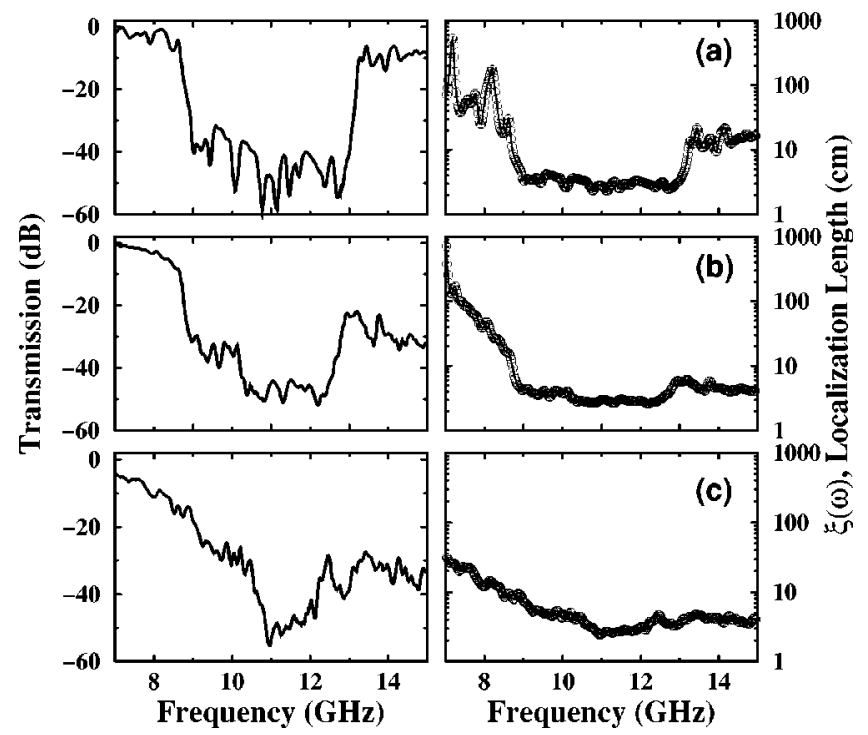

FIG. 2. [Left panel] The measured transmission spectra for (a) periodic $(r=0)$, and for disordered dielectric photonic crystals with (b) $r=a / 9$ and (c) $r=a / 4$. The photonic stop band shrank as the amount of disorder was increased. [Right panel] The localization length, $\xi(\omega)$, as a function of frequency for the corresponding photonic crystals. The localization length became smaller than the length of the crystal in the presence of disorder.

where $L=15.4 \mathrm{~cm}$ is the crystal length and $\langle\cdots\rangle$ denotes the ensemble averaging over various configurations. Figure 2 (right panel) displayed the calculated localization length by using Eq. (1) for (a) the periodic and (b), (c) the disordered cases. For the periodic crystal, while the localization lengths were much smaller than the length of the crystals for the frequencies within the photonic band gap, the localization lengths corresponding to lower and higher transmission bands were larger than the crystal length. On the other hand, the localization length became smaller as we increased the amount of the disorder in disordered crystals [see Fig. 2(c), right panel].

\section{B. Metallic photonic crystals}

Next, we performed the transmission measurements corresponding to the periodic and disordered metallic photonic crystals. The metallic photonic crystals were constructed from cylindrical copper rods with radius $1.45 \mathrm{~mm}$, and periodicity $a=1.1 \mathrm{~cm}$. Figure 3 (left panel) displays the measured transmission spectra as a function of frequency. For the periodic crystal [see Fig. 3(a), left panel], we were able to observe the upper band edge of the metallicity gap which extended from $0 \mathrm{GHz}$ to the plasma frequency $\omega_{p}$ $=11.67 \mathrm{GHz} .^{42,44,47}$ We also observed the photonic band gap that extended from 15.37 to $20.82 \mathrm{GHz}$ due to the periodicity of the crystal. ${ }^{44,46}$

When we introduced disorder to the metallic crystals, we observed two important behaviors. First, the metallicity gap still appeared in the transmission spectrum with a plasma frequency that was shifted to the lower frequencies. Second, the photonic band gap quickly disappeared. ${ }^{32}$ We also investigated how the localization length changed as a function of 


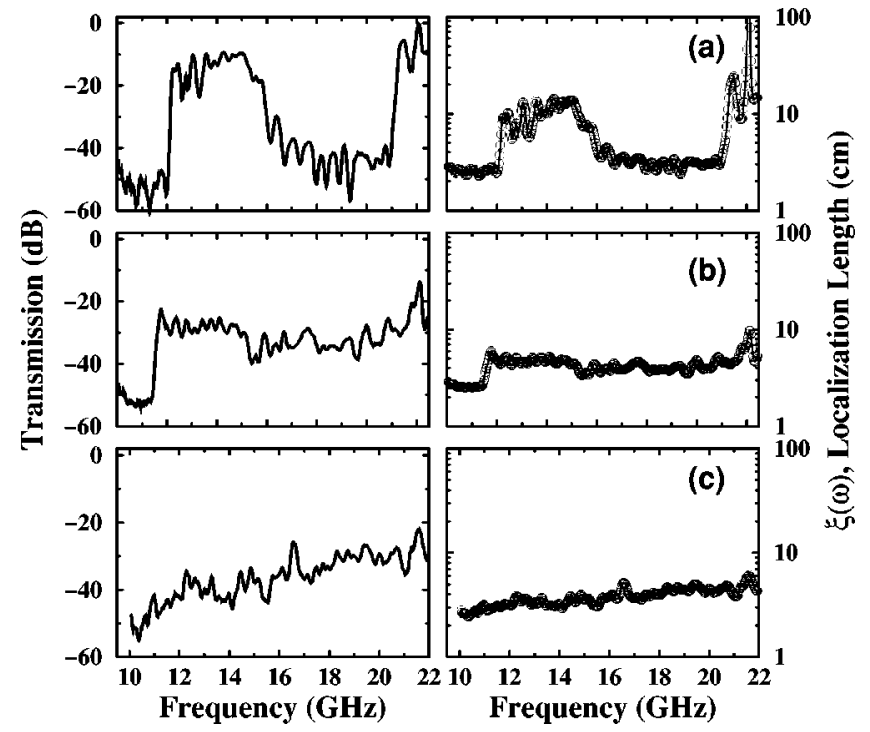

FIG. 3. [Left panel] The measured transmission spectra for (a) periodic, and for disordered metallic photonic crystals with (b) $r$ $=a / 9$ and (c) $r=a / 4$. As we increased the amount of disorder, the plasma frequency shifted to lower frequencies and the photonic band gap closed quickly. [Right panel] The localization length, $\xi(\omega)$, as a function of frequency for the corresponding metallic photonic crystals.

the disorder. The calculated localization length as a function of frequency is displayed in Fig. 3 (right panel). For the periodic crystal, the localization length for frequencies lying within the metallicity and photonic band gap were around 2 $\mathrm{cm}$ which was much smaller than the size of the crystal, i.e., $15.4 \mathrm{~cm}$. In the presence of disorder, $\xi$ had very small values. As an example, we obtained $\xi \sim 3 \mathrm{~cm}$ for moderately large disorder, i.e., $r=a / 4$.

The discrepancy between the behavior of the photonic band gap for the disordered dielectric and metallic crystals can be explained by the underlying mechanism responsible for the formation of the photonic band gap. As described in Ref. 35, the effect of disorder results in different changes to the system's properties. If the Bragg-like multiple scattering is the dominant one, the photonic band gaps should close quickly with increasing disorder. On the other hand, if the dominant mechanism is the Mie resonances, the photonic band gaps should survive even for large amounts of disorder, in a similar way that the electronic band gap survives in amorphous semiconductors. Under this explanation, we expect the dominant mechanism for the formation of the photonic band gap to be the Bragg scattering in metallic crystals, and the Mie resonances for the dielectric crystals.

\section{DEFECT CHARACTERISTICS IN THE PRESENCE OF DISORDER}

\section{A. Dielectric photonic crystals}

In literature, most of the photonic crystal based applications were built around the cavities which were formed by removing material from otherwise perfect PBG structures. Therefore, it is very important to investigate influence of

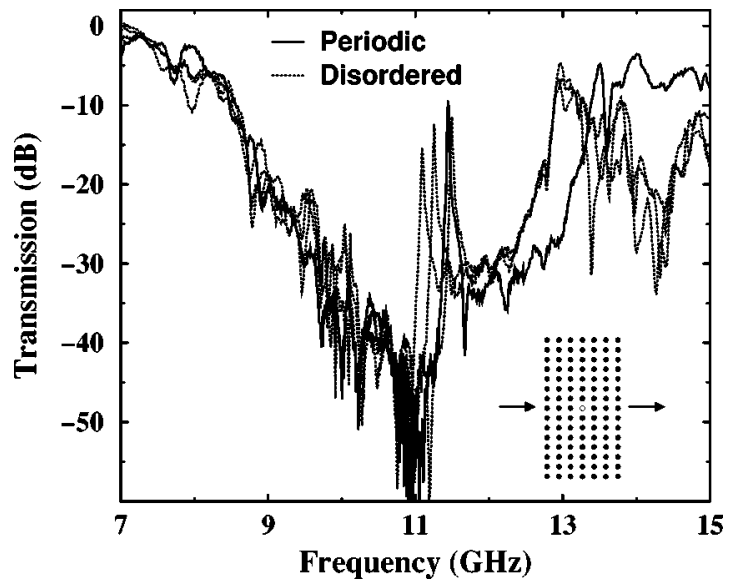

FIG. 4. The measured transmission spectra of single rod removed cavities in the periodic (solid line) and weakly disordered (dotted lines, $r=a / 9$ ) dielectric photonic crystals. Absence of translational symmetry leads to different defect modes for each defect in the disordered photonic crystals. Inset: Schematics [top view] of the cavity structure in the periodic crystal where $(O)$ symbol denotes the removed rod. For the disordered case, the rod was removed from the center, upper, and lower sites near to the center at each time.

disorder on the cavity characteristics. To address influence of the disorder on defect characteristics, we measured the transmission spectra of weakly disordered (with a disorder parameter $r=a / 9$ ) dielectric photonic crystals which consisted of $7 \times 15$ rods (see inset in Fig. 4).

We first measured the transmission spectrum of a cavity inside the perfect dielectric crystal. In order to generate a defect, we removed a single rod from the center of the crystal (see inset in Fig. 4). As shown in Fig. 4 (solid line), a localized cavity mode having a defect frequency $11.44 \mathrm{GHz}$ and a quality factor 530 appeared within the stop band.

For the disordered case $(r=a / 9)$, we performed the transmission measurements by removing a single rod from various locations for each time. First we generated a cavity at center of the crystal, then we formed cavities at upper and lower neighboring sites to the center. Due to absence of the translational symmetry, we observed three different cavity modes (dotted lines in Fig. 4) corresponding to each defect configuration. All of these modes had lower quality factors compared to the periodic case. For higher disorder values, we did not observe any defect modes within the stop band.

For the periodic case, the measured defect frequency and the quality factor were independent of the defect location. However, in the presence of positional disorder, depending on the size of the cavity, the resonance frequency shifted towards to lower or higher frequencies. Therefore, the cavity frequency depends on the position of the defect site in the disordered photonic crystals.

\section{B. Metallic photonic crystals}

Introducing metallic photonic crystals had certain advantages, namely, high rejection ratio, nearly perfect reflectivity at microwave frequencies, and large metallicity gap extend- 


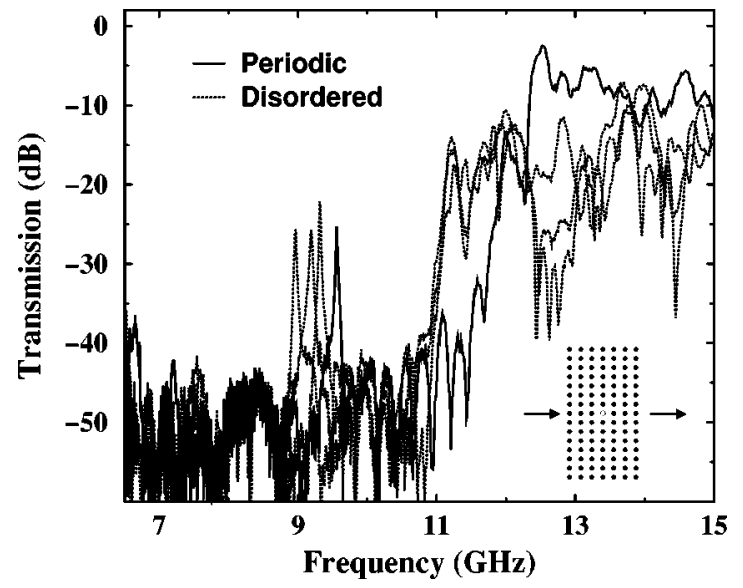

FIG. 5. The measured defect characteristics of periodic (solid line) and weakly disordered (dotted lines, $r=a / 9$ ) metallic photonic crystals with single removed rod. Highly confined cavity modes appeared within the metallicity gap. Inset: Schematics [top view] of the cavity structure in the periodic crystal where $(O)$ symbol denotes the removed rod. For the disordered case, the rod was removed from the center, upper, and lower sites near to the center at each time.

ing from zero frequency up to the plasma frequency. Therefore, for certain metallic cavity based applications, it is important to know how the defect frequency is affected by the presence of disorder in these structures.

In order to investigate the influence of disorder on the cavity frequency, we first measured the defect characteristics of periodic metallic photonic crystals by removing a single rod from the center of the crystal. Figure 5 (solid line) displays the transmission spectrum. We observed a defect mode within the metallicity gap with a frequency $9.56 \mathrm{GHz}$ and a quality factor 330 . It is important to note that the defect mode also appeared in the photonic band gap (data not shown in Fig. 5) with very low quality factor.

We also observed three distinct defect modes corresponding to cavities inside the disordered crystals (dotted lines in Fig. 5). It is also important to note that the plasma frequencies corresponding to disordered cases were shifted towards lower frequencies by $0.72 \mathrm{GHz}$ compared to the periodic case.

The variation of the defect frequencies within a certain frequency range was expected since the corresponding volume of the cavities were not identical to each other as in the periodic case. A larger defect volume leads to a lower defect frequency or a longer wavelength. Therefore, due to absence of the translational symmetry in the random crystal, it is possible to change the defect frequency by changing the position of the removed rod. This is similar to the tuning of the cavity frequency in quasiperiodic (Penrose crystal) dielectric photonic crystals. $^{48}$

\section{WAVE GUIDING THROUGH COUPLED CAVITIES AND EFFECTS OF DISORDER}

Recently, we have experimentally demonstrated another type of propagation mechanism for EM waves along coupled

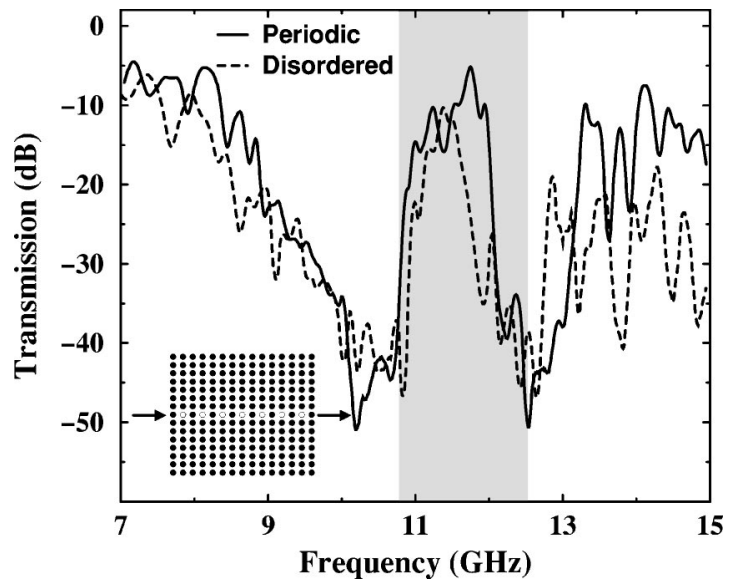

FIG. 6. Measured transmission through coupled cavities in twodimensional periodic (solid line) and weakly disordered (dotted line) dielectric photonic crystals. A waveguiding band (gray region) was formed due to coupling between localized defect modes. Inset: Schematic drawing of the coupled-cavity structure which was constructed by removing rods ( $\bigcirc$ symbols) with a periodicity of $2 a$.

cavities in 1D and 3D photonic band gap structures. ${ }^{13,25}$ The coupled-cavity waveguide (CCW) structures can be used in many photonic applications such as lossless and reflectionless waveguides, waveguide bends, ${ }^{13}$ EM-beam splitters, ${ }^{24,20}$ and dispersion compensators. Moreover, strong enhancement of spontaneous emission at the $\mathrm{CCW}$ band edges was reported. ${ }^{25}$

In this section, we investigate guiding and bending of EM waves through CCW's in 2D dielectric and metallic photonic crystals. We also address the influence of the weak disorder $(r=a / 9)$ on the guiding and bending in the CCW's. In our experiments, the CCW's were constructed by creating coupled defects in $15 \times 15$ square arrays of dielectric and metallic rods. Each defect was formed by removing a single rod from the crystal. In all cases, the straight and bended CCW's consisted of 7 coupled cavities with a periodicity of $2 a$ (see the insets in Figs. 6 and 7).

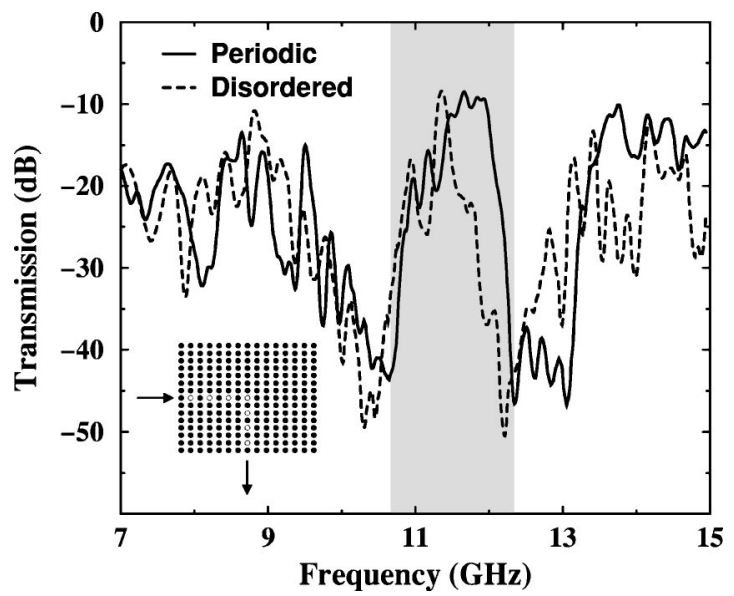

FIG. 7. Measured transmission through a $90^{\circ}$ bended CCW in two-dimensional periodic (solid line) and weakly disordered (dotted line) dielectric photonic crystals. Inset: Schematic drawing of the $\mathrm{CCW}$ bend structure where $(\bigcirc)$ symbol denotes the removed rod. 


\section{A. Dielectric photonic crystals}

We first measured the transmission spectra of the dielectric straight CCW's (see the inset in Fig. 6). For the periodic case, we observed a waveguiding band, or a defect band (gray region in Fig. 6) extending from 10.50 to $12.51 \mathrm{GHz}$. The bandwidth of the CCW's can be adjusted by changing the coupling strength between the localized cavity modes, i.e., decreasing the intercavity distance leads to a wider bandwidth. It is important to note that, as shown from Fig. 6, the defect band had very sharp band edges compared to the PBG edges, this property can be used to construct photonic switches. $^{24,20}$

Then we performed measurements on weakly disordered crystals with $r=a / 9$. In this case, the guiding of EM waves along the coupled cavities was also achieved (dotted line in Fig. 6) even if the waveguiding band significantly deformed for higher frequencies. Moreover, the resulting waveguiding band was narrower compared to the periodic CCW's.

The measured transmission characteristics of a $90^{\circ}$ bended $\mathrm{CCW}$ in periodic and disordered photonic crystals were presented in Fig. 7. Bending of the EM waves in the periodic crystal was achieved throughout the defect band (gray region in Fig. 7) that extended from 10.68 to 12.34 $\mathrm{GHz}$. However, the resulting bandwidth was narrower than the bandwidth that was observed for a straight CCW (Fig. 6). The bending efficiency ${ }^{49}$ was higher than $50 \%$ throughout the waveguiding band.

Although the introduction of weak disorder slightly narrowed the waveguiding band, we still observed high transmission of EM waves through the $90^{\circ}$ sharp bend (Fig. 7, dotted line). Similar to the periodic case, the disorder affects significantly the higher frequency side of the waveguiding band.

\section{B. Metallic photonic crystals}

We also investigated the waveguiding phenomena in $2 \mathrm{D}$ periodic and disordered metallic photonic crystals. Figure 8 displays the measured transmission spectra corresponding to periodic (solid line) and disordered (dotted line) straight CCW's. We observed two distinct guiding bands in metallic case. While the lower band appeared within the metallicity gap, the higher band appeared inside the photonic band gap. This result is expected since introduction of a defect into the metallic crystal leads to two distinct defect modes in the metallicity and photonic band gaps. As shown in Fig. 7, it was observed that bandwidth of the defect band in the metallicity gap is narrower than bandwidth of the defect band in the photonic band gap.

The introduction of disorder significantly affected the waveguiding properties of the metallic CCW's. As shown in Fig. 8, the disordered $\mathrm{CCW}$ structures had waveguiding bands with much lower transmission and bandwidths than the periodic metallic CCW structures. The defect band in the photonic band gap was significantly affected by the disorder.

Figure 9 shows the transmission characteristics of $90^{\circ}$ bended metallic CCW structures. We again observed two waveguiding bands in the metallicity and photonic band gap regions. However, the resulting bandwidths were narrower

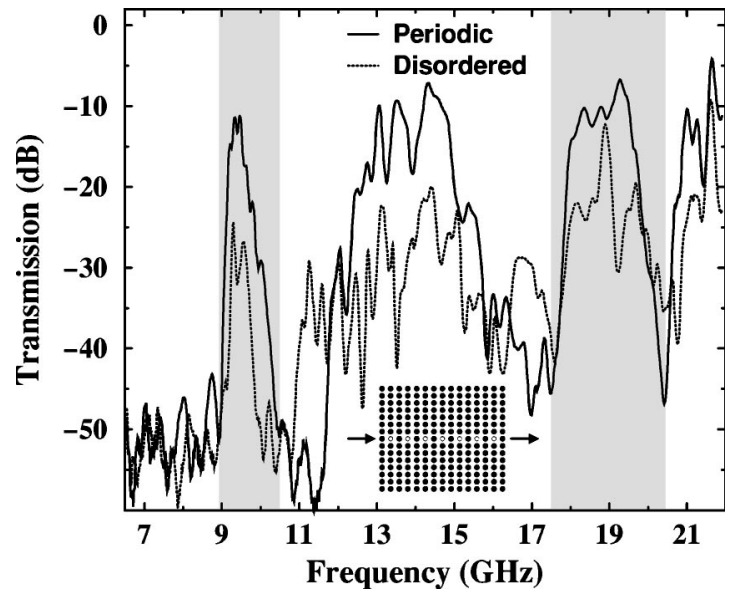

FIG. 8. Measured transmission through coupled cavities in twodimensional periodic (solid line) and weakly disordered (dotted line) metallic photonic crystals. Two waveguiding bands (gray regions) were formed within the metallicity gap and the photonic band gap due to coupling between localized defect modes. Inset: Schematics of the coupled-cavity structure which was constructed by removing rods ( $\bigcirc$ symbols) with a periodicity of $2 a$.

than the bandwidths observed for the straight metallic CCW's similar to what we observed in the dielectric photonic crystals. When we introduced the weak disorder, it was observed that the defect band in the photonic band gap disappeared.

Observation of guiding and bending of EM waves in 2D dielectric metallic CCW's has a technological importance. For instance, waveguides, waveguide bends, and splitters built around the coupled-cavity structures can be used to design 2D optical circuits. Since it is easy to fabricate the 2D CCW's structures at optical wavelengths.

It is important to note that the maximum transmission in the waveguiding band of the $2 \mathrm{D}$ photonic crystals did not reach unity in contrast to what we had observed in 3D photonic crystals. ${ }^{13}$ This can be explained by the absence of

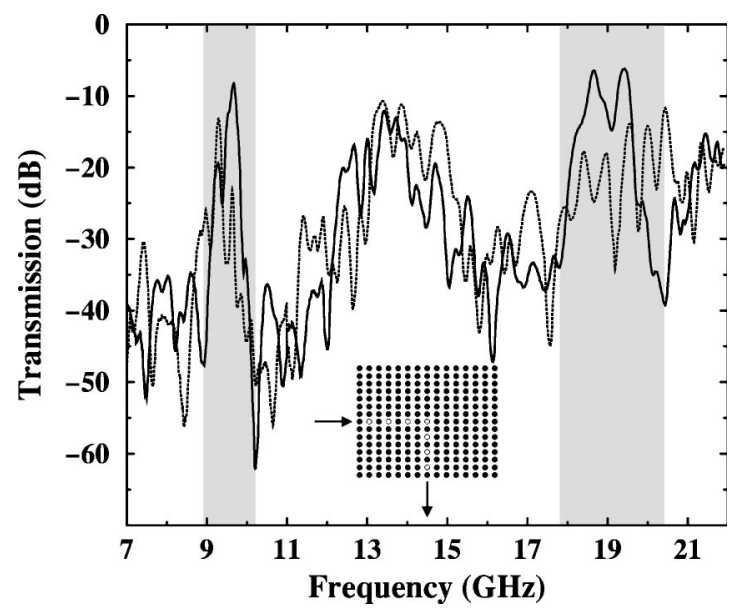

FIG. 9. Measured transmission through a $90^{\circ}$ bended CCW in two-dimensional periodic (solid line) and weakly disordered (dotted line) metallic photonic crystals. Inset: Schematics of the CCW bend where $(\bigcirc)$ symbol denotes the removed rod. 
confinement of the EM waves along the vertical direction (along the rod axis), ${ }^{50}$ that resulted in leakage of EM waves along this direction.

\section{CONCLUSIONS}

In conclusion, we investigated the influence of disorder on photonic band gap characteristics of 2D dielectric and metallic photonic crystals. We observed that positional disorder had a minor effect on the photonic band gap of the dielectric crystals. For the metallic crystals, the photonic band gap that originated from the periodicity quickly disappeared in the presence of disorder. However, the metallicity gap persisted even if a large amount of disorder was introduced to the crystals. We investigated effect of the disorder on defect characteristics. In disordered photonic crystals, in which the translational symmetry was absent, the defect frequency was changed depending on the position of the defect site. We also reported the observation of guiding and bending of EM waves through coupled cavities in dielectric and metallic crystals. Moreover, the influence of disorder on the waveguiding through the coupled-cavity waveguide structures was investigated.

\section{ACKNOWLEDGMENTS}

This work was supported by NATO Grant No. SfP971970, National Science Foundation Grant No. INT9820646, Turkish Department of Defense Grant No. KOBRA-001, and Thales JP8.04. Ames Laboratory is operated for the U.S Department of Energy by Iowa State University under Contract No. W-7405-Eng-82.
*Author to whom correspondence should be addressed. Electronic address: bayindir@fen.bilkent.edu.tr

${ }^{1}$ J. D. Joannopoulos, R. D. Meade, and J. N. Winn, Photonic Crystals: Molding the Flow of Light (Princeton University Press, Princeton, NJ, 1995).

${ }^{2}$ For a recent review, see articles in Photonic Crystal and Light Localization in the 21st Century, edited by C. M. Soukoulis (Kluwer, Dordrecht, 2001).

${ }^{3}$ K. M. Ho, C. T. Chan, and C. M. Soukoulis, Phys. Rev. Lett. 65 , 3152 (1990).

${ }^{4}$ S. John, Phys. Rev. Lett. 53, 2169 (1984); 58, 2486 (1987); Phys. Today 44, 32 (1991).

${ }^{5}$ E. Yablonovitch, T. J. Gmitter, R. D. Meade, A. M. Rappe, K. D. Brommer, and J. D. Joannopoulos, Phys. Rev. Lett. 67, 3380 (1991).

${ }^{6}$ S. John, in Photonic Band Gap Materials, edited by C. M. Soukoulis (Kluwer, Dortrecht, 1996), p. 563.

${ }^{7}$ E. Ozbay, G. Tuttle, M. M. Sigalas, C. M. Soukoulis, and K. M. Ho, Phys. Rev. B 51, 13961 (1995).

${ }^{8}$ A. Mekis, J. C. Chen, I. Kurland, S. Fan, P. R. Villeneuve, and J. D. Joannapoulos, Phys. Rev. Lett. 77, 3787 (1996).

${ }^{9}$ Shawn-Yu Lin, E. Chow, V. Hietala, P. R. Villeneuve, and J. D. Joannopoulos, Science 282, 274 (1998).

${ }^{10}$ B. Temelkuran and E. Ozbay, Appl. Phys. Lett. 74, 486 (1999).

${ }^{11}$ A. Chutinan and S. Noda, Phys. Rev. B 62, 4488 (2000).

${ }^{12}$ M. Loncar, D. Nedeljkovic, T. Doll, J. Vuckovic, A. Scherer, and T. P. Pearsall, Appl. Phys. Lett. 77, 1937 (2000).

${ }^{13}$ M. Bayindir, B. Temelkuran, and E. Ozbay, Phys. Rev. Lett. 84, 2140 (2000); Phys. Rev. B 61, R11 855 (2000).

${ }^{14}$ O. Painter, R. K. Lee, A. Scherer, A. Yariv, J. D. O’Brien, P. D. Dapkus, and I. Kim, Science 284, 1819 (1999).

${ }^{15}$ A. Mekis, M. Meier, A. Dodabalapur, R. E. Slusher, and J. D. Joannopoulos, Appl. Phys. A: Mater. Sci. Process. 69, 111 (1999).

${ }^{16}$ J. C. Knight, J. Broeng, T. A. Birks, and P. St. J. Russell, Science 282, 1476 (1998).

${ }^{17}$ M. Bayindir, B. Temelkuran, and E. Ozbay, Appl. Phys. Lett. 77, 3902 (2000).

${ }^{18}$ P. R. Villeneuve, D. A. Abrams, S. Fan, and J. D. Joannopoulos, Opt. Lett. 21, 2017 (1996).
${ }^{19}$ R. W. Ziolkowski and M. Tanaka, Opt. Quantum Electron. 31, 843 (1999)

${ }^{20}$ S. Lan, S. Nishikawa, and O. Wada, Appl. Phys. Lett. 78, 2101 (2001).

${ }^{21}$ B. Temelkuran, M. Bayindir, E. Ozbay, R. Biswas, M. M. Sigalas, G. Tuttle, and K. M. Ho, J. Appl. Phys. 87, 603 (2000).

${ }^{22}$ N. Stefanou and A. Modinos, Phys. Rev. B 57, 12127 (1998).

${ }^{23}$ A. Yariv, Y. Xu, R. K. Lee, and A. Scherer, Opt. Lett. 24, 711 (1999).

${ }^{24}$ M. Bayindir, S. Tanriseven, and E. Ozbay, Appl. Phys. A: Mater. Sci. Process. A72, 117 (2001); M. Bayindir, S. Tanriseven, A. Aydinli, and E. Ozbay, ibid. 73, 125 (2001).

${ }^{25}$ M. Bayindir, E. Ozbay, B. Temelkuran, M. M. Sigalas, C. M. Soukoulis, R. Biswas, and K. M. Ho, Phys. Rev. B 63, 081107(R) (2001).

${ }^{26}$ A. R. McGurn, K. T. Christensen, F. M. Mueller, and A. A. Maradudin, Phys. Rev. B 47, 13120 (1993).

${ }^{27}$ L. Pavesi and P. Dubos, Semicond. Sci. Technol. 12, 570 (1997).

${ }^{28}$ S. A. Bulgakov and M. Nieto-Vesperinas, J. Opt. Soc. Am. A 15, 503 (1998).

${ }^{29}$ A. R. McGurn, P. Sheng, and A. A. Maradudin, Opt. Commun. 91, 175 (1992).

${ }^{30}$ M. M. Sigalas, C. M. Soukoulis, E. N. Economou, C. T. Chan, and K. M. Ho, Phys. Rev. B 48, 14121 (1993).

${ }^{31}$ M. M. Sigalas, C. M. Soukoulis, C. T. Chan, and D. Turner, Phys. Rev. B 53, 8340 (1996).

${ }^{32}$ G. Guida, Opt. Commun. 156, 294 (1998).

${ }^{33}$ H. Y. Ryu, J. K. Hwang, and Y. H. Lee, Phys. Rev. B 59, 5463 (1999).

${ }^{34}$ A. A. Asatryan, P. A. Robinson, L. C. Botten, R. C. McPhedran, N. A. Nicorovici, and C. M. de Sterke, Phys. Rev. E 62, 5711 (2000).

${ }^{35}$ E. Lidorikis, M. M. Sigalas, E. N. Economou, and C. M. Soukoulis, Phys. Rev. B 61, 13458 (2000).

${ }^{36}$ G. Guida, T. Brillat, A. Ammouche, F. Gadot, A. De Lustrac, and A. Priou, J. Appl. Phys. 88, 4491 (2000).

${ }^{37}$ M. Stoytchev and A. Z. Genack, Phys. Rev. B 55, R8617 (1997).

${ }^{38}$ Yu. A. Vlasov, M. A. Kaliteevski, and V. V. Nikolaev, Phys. Rev. B 60, 1555 (1999).

${ }^{39}$ A. Chutinan and S. Noda, J. Opt. Soc. Am. B 16, 1398 (1999).

${ }^{40}$ M. M. Sigalas, C. M. Soukoulis, C. T. Chan, R. Biswas, and K. 
M. Ho, Phys. Rev. B 59, 12767 (1999).

${ }^{41}$ A. Modinos, V. Yannopapas, and N. Stefanou, Phys. Rev. B 61, 8099 (2000).

${ }^{42}$ D. R. Smith, S. Schultz, N. Kroll, M. M. Sigalas, K. M. Ho, and C. M. Soukoulis, Appl. Phys. Lett. 65, 645 (1994).

${ }^{43}$ M. M. Sigalas, C. T. Chan, K. M. Ho, and C. M. Soukoulis, Phys. Rev. B 52, 11744 (1995).

${ }^{44}$ D. F. Sievenpiper, M. E. Sickmiller, and E. Yablonovitch, Phys. Rev. Lett. 76, 2480 (1996).

${ }^{45}$ E. Ozbay, B. Temelkuran, M. M. Sigalas, G. Tuttle, C. M. Soukoulis, and K. M. Ho, Appl. Phys. Lett. 69, 3797 (1996).
${ }^{46}$ J. B. Pendry, A. J. Holden, D. J. Robbins, and W. J. Stewart, J. Phys.: Condens. Matter 10, 4785 (1998).

${ }^{47}$ B. Temelkuran, H. Altug, and E. Ozbay, IEE Proc.: Optoelectron. 145, 409 (1998).

${ }^{48}$ M. Bayindir, E. Cubukcu, I. Bulu, and E. Ozbay, Phys. Rev. B 63, 161104(R) (2001).

${ }^{49}$ The bending efficiency was obtained by normalizing the transmission spectrum through the bend to that of the straight waveguide.

${ }^{50}$ S. G. Johnson, P. R. Villeneuve, S. Fan, and J. D. Joannopoulos, Phys. Rev. B 62, 8212 (2000). 